\title{
Diálogos
}

Durante 2009 y después de tres años de operación, 0300TV tomó la forma de una editorial independiente dedicada a la arquitectura, que opera con registros audiovisuales, publicaciones, textos críticos, investigaciones, exposiciones y conferencias. Siendo actualmente dirigida por Felipe De Ferrari y Diego Grass, su compromiso con la disciplina los ha llevado a asumir encargos de distinto alcance y escala. Han realizado más de sesenta entrevistas inéditas, concentrándose en arquitectos que participan en la producción y discusión arquitectónica en Asia, Europa y América.

Esta sección de entrevistas, en colaboración con la revista $\mathbf{A R Q}$, pretende dar a conocer y publicar parte del material generado.

Andreas Ruby es crítico y teórico de la arquitectura. Estudió Historia del Arte en la Universität zu Köln, especializándose en Teoría e Historia de la Arquitectura en la Ecole Spéciale d'Architecture en París con Paul Virilio y en la Columbia University en Nueva York, junto a Bernard Tschumi. Entre 1999 y 2001 fue editor de la revista Daidalos. En 2001 crea Textbild junto a Ilka Ruby, produciendo ensayos, libros y revistas de arquitectura, curando exhibiciones y dando consultoría a arquitectos. Desde z002 es miembro del equipo editorial de la revista Werk, Bauen + Wohnen, desde 2006 es parte del Comité Consultor del Museo Alemán de Arquitectura y desde 200 ry es miembro del jurado del premio de Arquitectura Hans Schäfer Pri๘e. Ha enseñado Teoría y diseño de arquitectura en Kassel Universität, Cornell University y en la Ecole Polytechnique Féderale de Lausanne. Actualmente enseña en el programa Metropolis en la Universitat Politècnica de Catalunya en Barcelona. 
0300TV

En relación al estado actual de la disciplina y específicamente al papel de las publicaciones, tu trabajo como autor y crítico ocupa un lugar particular. Acerca de este punto, recuerdo cuando entrevistamos a Mark Wigley el año pasado y él mostró una posición bastante extrema sobre la presente situación de las publicaciones de arquitectura:

"Hoy, la principal función de las revistas es completamente conservadora: actúan haciendo registro y levantamiento para crear un banco de datos, de modo que los arquitectos y los estudiantes de arquitectura de todo el mundo manejen más o menos la misma información: y cuando todos tienen la misma información, todos se creen igualmente importantes. También actúan como un mecanismo policiaco, que monitorea el campo disciplinar para asegurar la ausencia de intrusos y vándalos". Si se comparte la percepción de Wigley no es dificil concluir que hoy la crítica sería inexistente y el panorama, banal y superficial. Siendo tú mismo parte del mundo de las publicaciones, ¿estás de acuerdo con lo que dice Wigley? ¿o consideras que su afirmación es más bien funcional, justamente para posicionar mejor el medio que él representa?

\section{Andreas Ruby}

Concuerdo parcialmente con Wigley: es un hecho que para tener un panorama saludable de publicaciones se requiere de ambos elementos. Necesitas primero de una fuente de información y luego desde allí el público obtiene lo que necesita: esa es su primera dimensión, la de servicio prestado. Podríamos decir que funciona como las noticias, ¿no? Los noticiarios que sintonizas por la noche para tener una idea de lo que está ocurriendo afuera... pero estas noticias por sí solas no son suficientes. Creo que las publicaciones tienen necesariamente un rol adicional; podríamos decir que él es establecer una agenda. No sólo deben decirte lo que ha pasado, sino también deben dar un idea de lo que podría pasar, o tal vez de lo que debería pasar. En ese sentido creo que Volume es un ejemplo interesante, porque han tratado de explorar lo que los arquitectos aún no están haciendo, al revés de la mayoría de las revistas de arquitectura que se dedican a mostrar sólo lo que los arquitectos ya han hecho. De todos modos, ambas visiones son necesarias: debes saber lo que está pasando y también deberías tener una idea de lo que no está pasando y debiera ocurrir.

\section{TV}

Sobre el mismo tema Beatriz Colomina dice que el aparato de difusión de la arquitectura es lo que en definitiva construye los objetos arquitectónicos. Dada la reciente proliferación de medios dedicados a la arquitectura, ¿cuál es tu opinión respecto al rol que las publicaciones de arquitectura debieran tener, y desde ahí, cuál es el principal objetivo de tu trabajo? ¿Aspiras a hacer teoria, promover, informar, ganarte la vida, o todas las anteriores?

$R$ : Creo que cualquier revista responsable, cualquier publicación que circule en sociedad, debería orientarse hacia los pedazos faltantes, a lo que no está siendo acogido o teniendo lugar. Y puedes ver que lo que abusivamente está teniendo lugar en las revistas de arquitectura es el comentario sobre edificios recién terminados, a modo de un monitoreo de la actividad constructiva. Y a mí me parece más bien que se trata de una actividad motivada por fines simbólicos, como una forma de asegurarle a la comunidad global de arquitectos y de la disciplina que las cosas van bien, que los edificios se construyen y que los arquitectos seguirán teniendo trabajo. Al mismo tiempo, esta tendencia reduce la arquitectura al ámbi to de la construcción: sólo las cosas construidas son consideradas piezas de arquitectura. Obviamente la arquitectura comprende un campo mucho más amplio: por ejemplo, también contiene las actividades que tienen que ver con la modelación del entorno físico y que son ejecutadas por no-arquitectos. Un ejemplo precioso para mí sería el siguiente: imagina cualquier número típico de una revista de arquitectura conocida y haz una segunda edición de ese número, simplemente re fotografiando todo con el lente de la cámara girado en $180^{\circ}$-es decir, no mirando hacia el edificio sino mirando desde el edificio-. Obtendrás una imagen del mundo que parece no existir en todo el espectro mediático de la arquitectura y que para mí representa algo del problema que intento describir. Debido al concentrado interés de las revistas en los productos de arquitectos, se falla estrepitosamen te en la reflexión sobre los matices del impacto que un edificio debe generar. Si se trata de un edificio cuya capacidad de cambiar el medio no es mucho más grande que su huella en el suelo-que es el rango que habitualmente describen las fotografías publicadas - estamos frente a un problema. La arquitectura necesita ser una influencia para la sociedad completa, necesita afectar mucho más que el área contenida en los límites del sitio. Sería fantástico hacer una revista de arquitectura que no trata de los edificios en sí mismos, sino sobre sus efectos.

En la discusión sobre los malls hay un término de optimización que se relaciona a esto: catchments area. Cada vez que hay que localizar un shopping mall, la idea es hacerlo de tal manera que se obtenga una gran catchments area; es decir, mayor área de influencia traducida en cierto volumen de público que verá cambiada su vida, directamente, con esta aparición. Esta capacidad de los edificios o de un conjunto de edificios de generar un área de impacto para mí es muy interesante.

En ese sentido y, volviendo a la idea de hacer lo que se echa en falta, probablemente hoy no se necesite tanto una revista de arquitectura como una revista de urbanismo. Pero claro, se trata de un ámbito menos iconográfico; no puede describirse fácilmente, porque no se materializa en la forma de un objeto sino en, podríamos decir, una condición de campo. ¡Y los campos son muy difíciles de fotografiar! Esto demuestra cómo la misma fotografía y sus medios condicionan finalmente la manera en que las revistas de arquitectura pueden mirar el mundo; en esa dirección, sería muy interesante pensar en las diferentes posibilidades de difundir y dar cuenta de la nueva arquitectura. Por ejemplo, sería posible hacer una revista de arquitectura que como único medio visual tuviera caricaturas: ejemplos ya hay varios. El arquitecto americano West Jones ha mostrado ya sus proyectos a través de una serie de caricaturas, tanto de edificios como de escenarios arquitectónicos; en la última Bienal de Venecia había una exposición sobre Dubai con el mismo recurso. En los dos casos la elección de un medio diferente hizo posible que muchos temas disímiles pudieran ponerse sobre la mesa.

\section{TV}

Respecto a la última Bienal de Venecia - "Architecture beyond buildings" (2008)allí se mostró una arquitectura difusa, un lenguaje más bien impreciso y un discurso lleno de cuestionamientos acerca de los límites de la disciplina. Cuando hablamos con Alejandro Aravena sobre la Bienal él sostuvo que Aaron Betsky se equivocaba al hablar de arquitectura más allá de los edificios y que en vez debió hablar de edificios más allá de la arquitectura. ¿Qué piensas de esa Bienal, del punto de vista de Betsky y de tu experiencia en el pabellón holandés?

$R$ : Cuando por primera vez escuché el lema de esa Bienal pensé que no era un mal comienzo. Lo entendí en el sentido de una imagen que

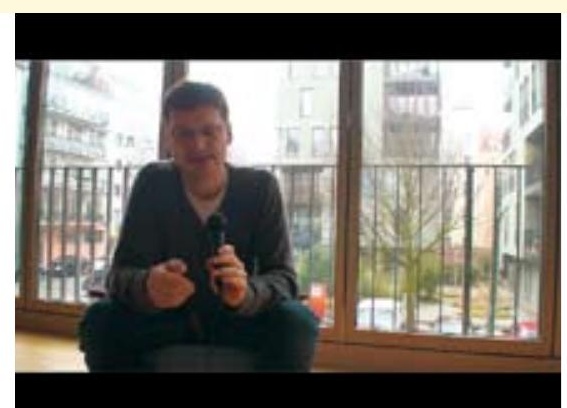


contiene una dirección: dice algo sobre lo que empieza aquí -en un punto conocido- y termina impredeciblemente en alguna otra parte. Entonces, cuando el comentario afirma arquitectura más allá de los edificios, podría haberse referido a una arquitectura que no se restringe sólo a los edificios, sino que tiene alg̊o así como un alcance mayor. Es muy simple y consistente; después de escuchar el título pensaba que sería una Bienal realmente interesante. Es una pena que Betsky haya comprometido de una manera tan extrema el potencial del tema. Si sólo recuerdas el montaje del Arsenale, que tradicionalmente ha sido una suerte de declara.ción de principios de cada curador de la Bienal, verás cómo la discusión se liquidaba, mientras que por el contrario el pabellón de Italia, por ejemplo, se planteaba de una manera coral y asistida.

En realidad Venecia no se trató de arquitectura más allá de los edificios: se trató de arquitectura reducida a objetos de star-architects y se convirtió en una especie de joyería de arquitectura contemporánea, muy pero muy desilusionadora. Y el hecho que el premio a la mejor intervención se lo hayan dado a Greg Lynn me pareció un poco ridículo, porque la pieza por la que fue premiado se siente, en mi opinión, más bien como un broma cínica. Por eso no entiendo realmente si el problema es que Betsky no captó el potencial de la tesis que se propuso presentar con el título de la Bienal, o tal vez el título fue en realidad la propuesta de un tercero y él realmente nunca quiso hacerlo.

Creo que el gran problema de la propuesta de Betsky en el Arsenale es que mostró un montón de arquitectos aclamados y consolidados que en sus inicios habían abordado la pregunta por la arquitectura más allá de los edificios. Un ejemplo podrían ser Coop-Himmelblau, quienes en el comienzo de su carrera habían trabajado mucho con la experiencia del espacio, aludiendo al espacio como la experiencia de una polvareda o de una explosión; era un tiempo en que ellos no construían y tenían el tiempo, el interés y la energía para experimentar realmente cómo la arquitectura podía existir en otros medios y materialidades que las de los edificios. Pero hoy Coop-Himmelblau se han convertido en una oficina proveedora de edificios a escala mundial, son el emblema de la vanguardia corporativa o de lo que se pudiera llamar así. Y no creo que ahora su investigación esté alineada en ningún punto con la capacidad de los edificios de generar espacios urbanos o de inspirar de alguna forma a la sociedad. Es puramente la producción de objetos atractivos, o no tan atractivos, a modo de fetiches. Y eso es exactamente lo contrario de lo que el tema de la Bienal sugería y, creo, es también lo contrario a lo que realmente necesitamos hoy.

Para fortuna de Betsky, la Bienal siempre contará con los pabellones nacionales y en a.gunos de ellos era posible encontrar buenos ejemplos de cómo este lema podía ser interpretado. Pienso en el de Bélgica, el de Japón y algunos otros. Eso compensó de alguna forma la sensación de oportunidad perdida que me pareció percibir y que intento describir.

\section{TV}

¿Qué personas a nivel global están haciendo trabajos que hoy te parezcan relevantes?

\section{$R:$ ¿Arquitectos, quieres decir?}

\section{TV}

No necesariamente; aprovecharía para am pliar al pregunta sobre obras o reflexiones que creas merezcan una segunda lectura, ¿qué cosas crees que deberíamos releer?

$R$ : Hablando de arquitectos, me gustan mucho los franceses Lacaton \& Vassal. Es una oficina que ha logrado levantar su propia agenda, en contrando un punto preciso para la inyección crítica; en su caso, es la crítica a la mal llamada mínima existencia, que es un término del modernismo, de los años treinta. Y ellos hacen notar que aún hoy gran parte de la vivienda colectiva, especialmente la vivienda social, está basada en esta especie de obituario que predefine cuánto espacio necesita un individuo para vivir. Pensando que la mayor disponibilidad de espacio podría enriquecer la calidad de vida, Lacaton \&e Vassal han conseguido establecer en cambio una aproximación al diseño de una casa que dobla o triplica los estándares típicos del metraje de la vivienda social, privilegiando el espacio antes que ciertas convenciones sobre la materialidad del proyecto.

Y especialmente me gusta el hecho que Lacaton \& Vassal tienen un entendimiento político de los que un arquitecto es. Tienen un interesante estudio llamado Place, que intenta delinear un tipo de actitud distinta para tratar ciertos proyectos de vivienda colectiva de los años sesenta y setenta en Francia, amenazados por un programa gubernamental de demolición a pesar de tratarse en su mayoría de departamentos aún habitados. Es un tipo de actividad que celebro en el trabajo de un arquitecto: estar interesado y habilitado no sólo para responder a las demandas de un programa o de un cliente, sino también en la detección de los problemas que necesitan abordarse hoy. Actualmente, creo que un arquitecto necesita más ser una persona que plantee preguntas y no sólo ser el agente que las res- ponda. Por eso para mí esta oficina francesa es muy interesante.

Un teórico que me gusta mucho y que ha sido muy influyente para mí es el arquitecto y crítico italiano Pier Vittorio Aureli. Me gusta porque tiene el coraje de emprender y llevar adelante una investigación y una pesquisa arquitectónica que parece estar completamente contra la corriente actual; diría que tiene la audacia de revisar conceptos del modernismo que aparecen profundamente comprometidos con su fracaso y trata de recuperar el potencial de los primeros modernos, como los experimentos urbanos de Hilberseimer, intentando actualizar esas ideas de acuerdo a la situación contemporánea. Para mí es un ejemplo de la posibilidad actual de definir un perfil arquitectónico que evite los clichés de la arquitectura de superestrellas -que sólo intenta producir objetos atractivos-y realmente operar desde una agenda programática, teórica; o sea, seguiría siendo posible hacer un buen trabajo pero no estar a la moda.

\section{TV}

Respecto a las ciudades, Alejandro Aravena decía que el desarrollo de la ciudad es el desarrollo de una red de oportunidades. A partir de 2007, y por primera vez en la historia de la humanidad, más de la mitad de la población del mundo vive en ciudades. Sobre este punto tengo dos preguntas: ¿Cómo entiendes actualmente los conceptos de centro y periferia? Y en segundo lugar, ¿ves reales diferencias entre la producción arquitectónica que proviene de la periferia en relación a la de los centros? Claro, no hablo sólo de diferencias económicas.

$R$ : Creo que el modelo de ciudad y suburbio, más bien de centro y suburbio, en el futuro no será sostenible. Básicamente porque parte del supuesto de crecimiento ilimitado de la ciudad, pero manteniendo una relación de jerarquías un poco perversa con el centro, que siempre se considera más valioso o importante. Y mientras más en el borde, el valor del espacio urbano sucumbe si se compara con el del núcleo; eso produce toda clase de problemas estructurales, partiendo por la movilidad y el tráfico. Ahora, es interesante ver que en ciudades como Los Ángeles, cuya realidad actual fue generada por las lógicas de una expansión desbordada, la gente ya no vive más en función del centro; tienden a crear en cambio pequeñas unidades urbanas dentro de la ciudad, que escapan al sistema de las jerarquías y que son equivalentes unas a otras; la dicotomía entre centro y periferia se transforma en relaciones urbanas polinucleares, algo que Mathias Ungers llamó en los años setenta ciudad archi- 
piélago. O sea, un sistema compuesto de islas, por así decirlo, con áreas vacías entre ellas. Para mí ese es un concepto mucho más prometedor a la hora de imaginar cómo organizar el crecimiento urbano hoy; los suizos lo han llamado ciudad-red, donde en vez de tener un campo urbano continuo, sin intensidades, se tiene un campo discontinuo de áreas densas separadas unas de otras por áreas completamente no-urbanas. Simplemente, en sí mismas la separación y la concentración en cada núcleo activo pueden crear intensidades que promueven otras dinámicas de intercambio. Como decía, la gente en Los Ángeles ya empezó a vivir la ciudad de este modo. Tal vez hace diez años - recuerdo haber visitado entonces la ciudad por primera vez para ver unos amigos- aún era posible encontrar personas que recorrían grandes distancias para asistir a una conferencia o para encontrarse con alguien en un café. Eso es rarísimo hoy en día; la congestión del tránsito se ha vuelto tan severa que es virtualmente imposible invertir una hora de congestión para llegar a un lugar, hacer algo ahí y luego ocupar otra hora completa en regresar a casa. Lo que pasó es que los ciudadanos comenzaron a consolidar sus actividades en sus propios barrios, generando algo muy parecido a la dinámica de los archipiélagos; cuando esto ocurre, significa que quizás ya no es necesario ni sano tener una alfombra urbana continua y que es posible manejar un sistema de entidades urbanas separadas, que se alejan de la idea de satélites en torno a un núcleo sino que son más bien equivalentes, como islas en un archipiélago.

\section{TV}

En una entrevista a Charles Jencks publicada en Volume, él habla de Gehry y de su desinterés en la generación de cambios... o del poder que ha alcanzado sin necesariamente tener una orientación para usarlo, a diferencia de Alberti o Palladio. También habla del poder de Koolhaas, pero parece escéptico de su capacidad actual de provocar cambios reales. Desde tu punto de vista, ¿quiénes son los actuales agentes de cambio?, ¿quién estaría en situación de definir la agenda disciplinar para los próximos años? Tal vez sea muy prematuro hablar de esto, pero ya nombraste a Anne Lacaton y Jean Philippe Vassal... ¿ellos podrían ser posibles candidatos?

R: Estaría de acuerdo con Jencks en que la arquitectura no tiene un punto de partida como podría tener una película... respecto a quién detenta el poder y quién además lo usa, por ejemplo, para replantear las condiciones de un encargo. Es como Roger Moore y James
Bond. Cuando Moore comenzó a interpretar al agente 00\%, evidentemente estaba heredando el rol de Sean Connery, conocido por la interpretación de actitudes machistas con las mujeres; por ejemplo, a Bond le parecía posible golpear a una mujer si ella no entraba en razón por las buenas y sus primeras películas contienen varios ejemplos de violencia aplicada a mujeres, como algo de lo más habitual. Y cuando Moore comienza a interpretar a Bond le dice a los productores que no está de acuerdo con ese elemento del personaje y que no lo quiere perpetuar: básicamente, cambia el rol. Entonces Moore cambia el curso de los hechos y no se limita a tomar el guión y ejecutarlo siguiendo lo que estaba establecido -el guión para estos efectos es casi como un programa arquitectónico-; al contrario, hace una relectura crítica y dice, "sabes, no estoy de acuerdo con esto y quiero hacerlo de otra manera".

Y no veo realmente a los arquitectos teniendo ese tipo de desobediencia civil, especialmente a los star-architects quienes podrían atreverse a actuar de una manera menos complaciente. Ellos podrían ajustar los programas, podrían cambiar las expectativas del cliente y efectivamente son los que están en condiciones de rechazar un proyecto cuando parezca adecua do hacerlo y ejercer así cierto poder. Para mí, hasta que veamos arquitectos que aprendan a ejercer esa influencia, diría que las figuras con el potencial de ser agentes de cambio son principalmente los políticos. Y es interesante desde Europa mirar continentes como Sudamérica, donde hay alcaldes políticos que tienen un tipo de poder muy especial y una manera extremadamente interesante de usar ese poder en la toma de decisiones. Y se trata de decisiones que ningún alcalde ni presidente europeo tomaría jamás. Enrique Peñaloza, el ex alcalde de Bogotá, es un ejemplo de lo que estoy hablando: él, contra todas las demandas de la clase alta de su ciudad, decidió cancelar la construcción de una serie de autopistas y, en cambio, empleó los mismos recursos para implementar toda una red peatonal y de ciclovías que atraviesa la ciudad en varias direcciones cambiando completamente la calidad del tejido urbano y la situación urbanística de la capital colombiana. Es crucial entender el impacto que tienen las decisiones sobre la infraestructura del trasporte público: se trata de asuntos estructurales que pueden sentar las bases para sucesivas intervenciones en la ciudad. Por ejemplo, a lo largo de estos paseos peatonales en Bogotá podría haber desarrollos de vivienda, con comercio local que podría activar los paseos; todo ello es evidentemente imposible si tienes autopistas, donde sólo hay lugar para el desplazamiento continuo y nadie puede parar. Para mí se trata de un tipo de trabajo generativo, que ante todo define prioridades y luego intenta fijar una agenda que permite implementar esa visión; hoy pareciera ser un trabajo más fácil de hacer para un político que para un arquitecto, pero aún así creo que los arquitectos tenemos la responsabilidad de recordarles a los clientes la necesidad de establecer prioridades. Entonces si un cliente propone un listado de demandas y requerimientos para un proyecto que según tu punto de vista está desajustado a la realidad o a la situación proyectual, por que atienden asuntos del corto plazo pero olvidan los aspectos del largo plazo, como arquitecto tienes la responsabilidad de hacerle notar su falta de visión. Y me temo que poquísimos arquitectos ejecutan esa práctica.

Hay un ejemplo muy bueno, en una escala pequeña, de este tipo de comportamiento que echo de menos en el manejo que el arquitecto podría hacer en la relación con los clientes. Un arquitecto debía proyectar una pequeña casa para una pareja; en cada reunión con ellos se enfrentaba a su imposibilidad para lograr acuerdos respecto a cómo debía ser la casa; era patente que había desavenencias de fondo en el matrimonio, hasta que un día el arquitecto los enfrentó y les dijo: "¿Saben? Ustedes no necesitan un arquitecto, necesitan un abogado y un divorcio".

Para mí esa reacción es un acto mucho más arquitectónico que el otro camino: ignorar el hecho que no se llevan bien y que no pueden llegar a un acuerdo sobre una idea para el proyecto, para luego construir la casa a modo de hecho consumado; se ha olvidado la alternativa de decir "no, ustedes no están listos para construir una casa, deben reconstruir su relación". Podrías ejercer ese mismo tipo de desobediencia civil en todos los encargos que recibas, como Lacaton \& Vassal hicieron con el encargo para renovar una pequeña plaza en Francia.

\section{TV}

¿Place d'Aucot?

$R$ : Claro, Place d' Aucot. Ahí ellos establecen que no era necesario intervenir el lugar para hacerlo más agradable, porque ya era agradable y era justamente lo que la gente necesitaba como espacio público, por lo que no hubo una intervención física sino recomendaciones de mantenimiento y gestión. Y me sorprende que la mayoría de los arquitectos aún se consideran como proveedores de un servicio y, bueno, creen que deben limitarse a hacer lo que el cliente quiere.

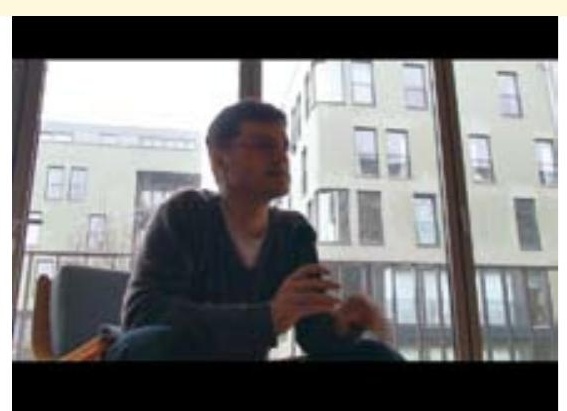




\section{TV}

Acerca de tu trabajo en Textbild y Ruby Press, específicamente sobre la ecléctica producción editorial de Textbild-que incluye desde libros sobre Zaha Hadid hasta Urban Transformations- me gustaría saber sobre la elección de los temas de los libros que editas. ¿Hay alguna metodologia?, ¿priman las intuiciones, las relaciones personales o las circunstancias? ¿O es que los libros que produces más bien evitan cualquier tipo de asociación?

R: Comenzamos haciendo libros básicamente recibiendo encargos de otras editoriales, que nos pedían editar un proyecto o contribuir en un libro determinado. Con los años nos hemos liberado cada vez más de este tipo de encargos para desarrollar nuestra propia agenda sobre las publicaciones que nos interesan, hasta la fundación de nuestra editorial el año pasado. La idea detrás de esto es llegar a hacer el tipo de libro que no está disponible, en lugar de clonar tipologías editoriales que ya están por todos lados; visto así, nuestro trabajo editorial podría resumirse en tres clases de libros que nos hemos propuesto hacer.

El primer tipo es el libro temático, en el que definimos un tema que nos parece importante seguir y estudiar; Urban Transformations, nuestro primer libro, pertenece a este tipo. Con él intentamos hacer una especie de antología, una obra coral donde invitamos a muchos autores a crear pequeños focos de atención en torno a un tema que, desde nuestro punto de vista, no ha sido explorado suficientemente.

El segundo tipo es la monografía arquitectónico-constructiva: es un tipo de libro que prácticamente se extinguió después de una época en que hubo buenísimos ejemplos. Curiosamente, este es uno de los libros donde uno esperaría encontrar una importante cantidad de información planimétrica, cortes, detalles y plantas que hoy han sido apartados por la mayoría de los editores a favor de las fotografías y los renders. Para mí esta tendencia muestra un empobrecimiento en la manera de plantear un libro de arquitectura y estoy ansioso por explorar ese nicho y estudiar un edificio realmente en detalle; yo mismo aprendí enormemente de ese tipo de publicaciones y por lo mismo estoy entusiasmadísimo por iniciar esta línea.

El tercer tipo de libro que nos interesa es un libro sobre la vivencia de una ciudad, vista. desde la óptica de un individuo particular. Se trata de replicar una experiencia que me gusta mucho y que es casi lo mejor que te puede pasar cuando visitas una ciudad por primera vez: tienes un amigo que vive ahí hace un tiempo. Es un lujo tremendo, un gran regalo si esa persona puede dedicarte algo de su tiempo y mostrarte la ciudad a través de sus propias experiencias, llevándote a lugares donde probablemente nunca habrías ido usando una guía turística. Son lugares que tal vez no tienen grandes cualidades desde el punto de vista arquitectónico en tanto edificios, pero generalmente tienen alguna calidad espacial, como el mercado nocturno de Kuala Lumpur que visitas a las 3 de la mañana. Creo que a través de este tipo de introducción personal estableces una relación emocional con el lugar que nunca podrías alcanzar como turista; para mí es fundamental al menos intentar traspasar la barrera del turismo cuando visitas una ciudad, coqueteando con ella como si fueses un local pero con ayuda de un verdadero local. Se trata de un conocimiento de lo urbano que explora otras áreas que se han dejado de lado; sería un buen concepto para una colección, contactar a una personalidad de una ciudad específica -quien tal vez no tenga que ver necesariamente con la arquitectura ni con el urbanismo pero que sí tiene una intensa relación con la ciudad - e intentar compartir, tomar ventaja de su experiencia con la ciudad para construir nuevas experiencias. Sería una empresa doble, pues no sólo tendríamos el retrato de una ciudad: también aparecería el retrato de una persona.

\section{TV}

Dijiste en algún momento que Rem Koolhaas alcanzó cierta influencia política usando la arquitectura como un medio de comunicación masivo. Me gustaría hacerte la misma pregunta que hiciste a Koolhaas alguna vez: ¿Cómo ves el rol del arquitecto en un futuro dominado por mandantes con poder absoluto y promotores de la construcción?

$R$ : En el mundo actual el arquitecto tiene una posición distinta a otras épocas, pero que nos recuerda ciertos aspectos constantes presentes en siglos anteriores, a lo largo de la historia de la arquitectura. Los arquitectos ya han tenido que someterse en ciertos niveles al poder absoluto y paralelamente han intentado lograr ciertos objetivos que, podríamos decir, pertenecen a una agenda oculta, a intereses que sólo incumben a la propia disciplina de la arquitectura. Creo que es fundamental aceptar esta condición y aprender a negociar con las entidades del poder. No podemos rechazar el poder, necesitamos trabajar con él. No diría que los arquitectos no deben trabajar cooperativamente, pero sí diría que deben hacer valer su peso e influencia. Después de todo, un tra- bajo cooperativo necesita a.l arquitecto del mismo modo en que los gobernantes necesitan a.l arquitecto. En la historia de la arquitectura se puede aprender mucho sobre cómo famosos arquitectos intentaron usar su capacidad para negociar entre las cosas que estaban obligados a hacer, aquellas que escapaban a su dominio y las que podrían ocurrir si ellos tomaban el control. Pero todo este proceso necesita de un entendimiento profundo del papel del arquitecto a partir de la posibilidad de la desobediencia civil: es decir, que el arquitecto nunca es solamente el encargado de satisfacer las necesidades de su cliente, pero sí siempre es responsable de defender el interés común. Y es la cosa más difícil, esta posición en la que estará el arquitecto mañana y también durante los próximos años; por definición su terreno estará en los conflictos de interés, articulando por una parte el interés individual y por otra el interés colectivo.

\section{TV}

Me gustaría pensar en las ambiciones del proyecto Urban Transformations primero y luego en su alcance real. ¿Cuál crees tú es el público que definitivamente va a consumir este libro? ¿Su objetivo último son los arquitectos, o se trata de un libro destinado a impactar en el mundo de los no arquitectos?

$R$ : Juzgando por la recepción que ha tenido el libro, podría decir que muchas personas que trabajan en instituciones gubernamentales se han interesado en él, porque les permite ver frentes de intervención donde ciertas acciones pueden y deben emprenderse, frentes en los que deben trabajar con arquitectos si se quiere producir algún cambio real. Creo que actualmente la arquitectura tiene una posibilidad de adquirir peso político, mientras logre comunicar a los políticos que puede ayudarlos a mejorar el entorno físico y social. Obviamente tanto arquitectos como políticos usan lenguajes diferentes, pero es posible producir cercanías... pienso en el famoso proyecto Elemental de Alejandro Aravena en Iquique. Es un ejemplo exacto donde eso fue lo que pasó: existía un programa de gobierno para subvencionar la producción de vivienda social y por otro lado una visión arquitectónica sobre cómo usar esos subsidios de una manera más inteligente. Y en definitiva produce un prototipo de vivienda que podría luego servir como modelo para otros tipos de edificios y además, podría servir como antecedente para una colaboración productiva, a nivel legislativo, entre los arquitectos y las instituciones gubernamentales, donde en realidad se trata de definir nuevos estándares para la producción 
del entorno construido. Estaríamos de lo más contentos si en realidad este libro pudiese atender y estimular esta clase de colaboración entre arquitectura y urbanismo o, más bien, entre planificadores, legisladores y políticos.

\section{TV}

Sobre el mismo tema... y te lo pregunto porque me parece crucial lidiar con los problemas reales... aunque la arquitectura no siempre parece suficiente para manejar estas preocupaciones, a la manera de la investigación de Lacaton \& Vassal sobre componentes políticos o burocráticos. ¿Estamos los arquitectos hoy en posición de negociar con la administración pública y privada? ¿O Elemental es más bien la excepción a la regla?

$R$ : Creo que cada día hay más arquitectos en posición de participar en esa discusión. Y lo que me parece más importante, hay más arquitectos interesados en hacerlo. Me parece que esa es una tremenda prioridad y aún muchos arquitectos no están interesados en concebir su trabajo como parte de la cadena de transformación del entorno físico y social, que excede el diseño de espacios. Para mí el diseño es parte de esa cadena pero no es su elemento medular; creo que los arquitectos necesitamos aprender a comunicarnos con los políticos y los economistas, por ejemplo, si queremos ser relevantes. Pienso que hoy un arquitecto es irrelevante si la sociedad sólo puede considerar su trabajo en términos estéticos; y ese es el caso de mucha de la arquitectura actual.

\section{TV}

Cuando usas como referencia Urban

Transformations... un libro de 400 páginas, 56 autores, 36 ilustraciones, 5 colores, ¿estos números constituirían una especie de manifiesto, un perfil deseable de la disciplina, una declaración respecto al escenario de la arquitectura hoy? Básicamente apareces como el moderador de una red muy amplia, una red que incluye el proyecto para el Parque de la Aguada en Santiago presentado por un texto de José Rosas y Pablo Allard. ¿Cómo te contactaste con ese caso específico?

$R$ : La idea de trabajar con muchos autores para este libro vino de un Congreso que alimentó el proyecto y que básicamente lo hizo posible -el II Congreso para la Construcción Sustentable en Shanghai en 2007 - donde la naturaleza propia de la reunión involucraba una convocatoria muy amplia que trajo voces de los cinco continentes. Es fundamental estudiar los temas urbanos desde una perspectiva regional, ya que por las diferentes condiciones locales se trata de una materia imposible de tratar desde un punto de vista genérico o a través de objetivos genéricos. De ahí heredamos el hecho de contar con múltiples autores, lo que nos pareció muy atractivo porque las contribuciones al Congreso eran un enorme campo desde donde seleccionar las discusiones más interesantes; en definitiva ese campo aportó casi el 50\% del libro. La otra mitad la completamos con personas que conocíamos previamente y cuyo trabajo nos parecía importante de incluir. Sin una red de muchas personas que a su vez conocen a muchas otras personas este libro no habría sido posible; de hecho creo que un libro como este no debiera hacerse sin esa red: me gusta esa visión caleidoscópica, con todos estos diferentes ángulos de lo que es una ciudad hoy, enunciados de primera mano. Creo que ese panorama tan genuino sería imposible de obtener a través de un solo autor, porque la pregunta por lo que es hoy una ciudad es muy difícil de responder. Entendimos que sólo es posible contestarla parcialmente, sólo desde la visión de una ciudad en particular; dime qué es lo urbano para ti y te diré de qué ciudad vienes. Entonces, en cada intento por describir la urbanidad hay un reporte de una cultura. urbana específica y por lo mismo era fundamental contar con muchas contribuciones para el libro.

\section{TV}

Finalmente, ¿Podrías describir los principales temas de la agenda futura de Textbild?

$R$ : Como te decía antes, nos concentraremos en editar usando esos tres tipos de vectores. Estamos interesados en contribuir a la construcción de una nueva agenda para la arquitectura, considerando qué puede hacer un arquitecto ahora, tras el colapso del starsystem, cosa que se podría establecer a partir de dos hechos: la crisis financiera de 2008 y la última Bienal de Venecia, que para mí representa el ocaso del estrellato en la arquitectura contemporánea. Con todos estos acontecimientos, habría que preguntarse una vez más por las prioridades y los frentes que la arquitectura contemporánea debiera ponerse por delante. ¿Cuál es el conjunto de estrategias, contenidos e ideas con los que la disciplina debiera comprometerse? Creo que en Textbild intentaremos producir simposios, exposiciones y más libros, para contribuir a nutrir esa búsqueda. En realidad creo que los arquitectos sentimos ahora una especie de vacío; parecier que por mucho tiempo nuestra única meta fue adquirir cierta destreza, o experiencia, en el diseño de formas exquisitas, lo que nos dejaba calificados, por ejemplo, para diseñar el Museo
Guggenheim. Ese camino parecía la definición normal del destino de un arquitecto: cada vez, hacer el edificio más espectacular posible para cualquier lugar en el mundo; hoy pareciera. que tenemos demasiados de esos edificios y no parecen plantear ninguna pregunta a nuestra sociedad, ni tampoco abordar las cuestiones que hoy surgen como tareas transversales. Creo que necesitamos redefinir qué es realmente hoy día la misión del arquitecto. De un modo interesante, me parece que estamos ante una situación similar a la que describía Jean Baudelaire en 1968: ¿qué podemos hacer tras la orgía? Refiriéndose al desenfreno de las revueltas estudiantiles, escribe creo que a inicios de los setenta este texto que describe la sensación de no saber ni dónde ir ni qué hacer, a pesar de la urgencia cierta de que había que hacer algo. Creo que ahí estamos, en esta especie de burbuja que nos priva de entender realmente lo que está pasando, pero que al mismo tiempo nos deja entrever que algo está profundamente equivocado de manera que sentimos que habría que intentar la definición de una agenda que indique cuál es el paso siguiente. Este presente tan particular que nos toca vivir para nosotros es un momento muy emocionante. En realidad, nos importa muchísimo participar de este proceso de búsqueda y redefiniciones de la agenda para la arquitectura actual. ARQ

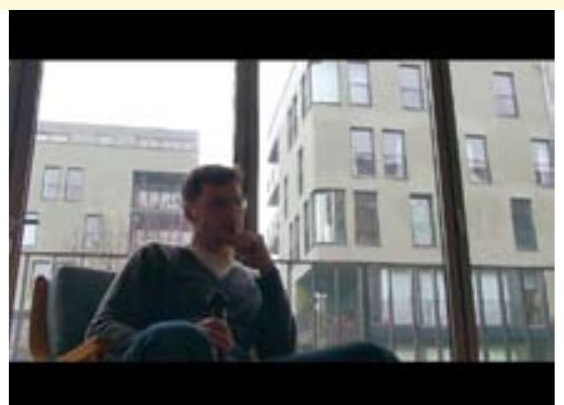

